\title{
Frequência de Acidente de uma Instalação Industrial com um Canal de Proteção Sujeito a Envelhecimento
}

\author{
Lucas G. Oliveira ${ }^{1}$ \\ Departamento de Engenharia Nuclear, Escola Politécnica, UFRJ, Rio de Janeiro, RJ \\ Danielle G. Teixeira ${ }^{2}$ \\ Programa de Engenharia Nuclear, COPPE, UFRJ, Rio de Janeiro, RJ \\ Paulo Fernando F. Frutuoso e Melo ${ }^{3}$ \\ Programa de Engenharia Nuclear, COPPE, UFRJ, Rio de Janeiro, RJ
}

\begin{abstract}
Resumo
Analisa-se a confiabilidade de sistemas de proteção de instalações industriais para o caso de equipamentos sujeitos a envelhecimento, importante na extensão da vida útil qualificada das instalações. Por meio do método das variáveis suplementares, desenvolveu-se um sistema de equações integro-diferenciais parciais e ordinárias acopladas para as probabilidades de um sistema de proteção de um canal sujeito a envelhecimento, que foi resolvido por diferenças finitas. O modelo foi validado pela comparação com resultados de canais com tempos de falha exponenciais. O método das variáveis suplementares exibe resultados razoáveis para valores de atributos de confiabilidade típicos das instalações industriais.
\end{abstract}

Palavras-chave. Frequência de Ocorrência de Acidentes, Envelhecimento, Cadeias de Markov, Variáveis Suplementares, Diferenças Finitas, Sistemas de Proteção, Instalações Industriais.

\section{Introdução}

A análise da confiabilidade de componentes de sistemas de proteção é de suma importância $[1,2]$. Mas, é necessário abordar os componentes que não se encontram mais em seu período de vida útil, ou seja, que estão envelhecendo. O propósito é o de permitir estabelecer políticas de inspeção e manutenção apropriadas que reduzam o custo e o tempo de parada da planta.

Neste caso, os tempos de falha não seguem mais distribuições exponenciais, o que implica na utilização de modelos com taxas de falha crescentes para representá-los, como as distribuições de Weibull ou lognormal [3], e o processo se torna não-markoviano [3]. Devem-se buscar alternativas. Nos métodos que utilizam processos estocásticos, um se

\footnotetext{
${ }^{1}$ giehllucas@poli.ufrj.br

22dteixeira@con.ufrj.br

${ }^{3}$ frutuoso@con.ufrj.br
} 
destaca: o das variáveis suplementares [3]. Este tem como vantagem a possibilidade de se gerar uma solução exata para o problema, descontando-se o erro numérico intrínseco e, não necessitando de métodos de otimização, como o dos estágios [3].

O objetivo deste trabalho é o de usar o método das variáveis suplementares para obter a frequência de acidente de uma instalação industrial equipada com um sistema de proteção constituído de um único canal, considerando o seu envelhecimento.

Para os sistemas de proteção, o parâmetro de confiabilidade de interesse é a sua indisponibilidade média, $\mathrm{U}$, que depende das taxas de falha, $\lambda$, e de reparo, $\mu$, do número de canais que o constituem, além da taxa de demanda $\nu$ e também das políticas de teste e manutenção e da sua lógica de atuação dos mesmos. O atributo que se considera é a frequência de ocorrência de acidentes, $\eta[1]$, definida como:

$$
\eta=\nu U(\lambda, \mu, \nu)
$$

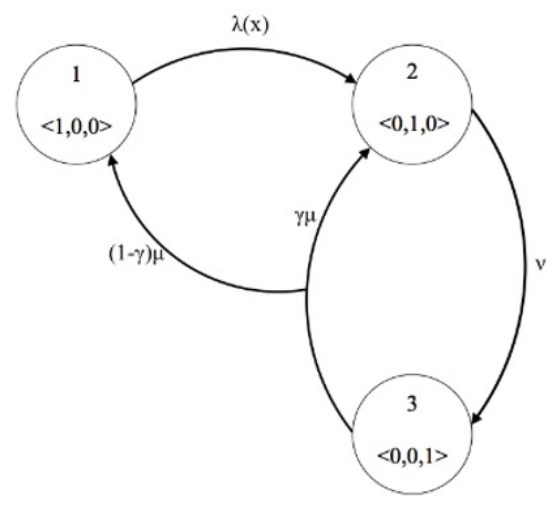

Figura 1: Diagrama de transição de estados para um canal envelhecendo

Considerando que um número inteiro de intervalos de teste é realizado num período de um ano, a frequência de ocorrência de acidentes será dada por [1]:

$$
\eta=\frac{\nu}{\tau_{p}} \int_{0}^{\tau_{p}} P_{2}(t) d t
$$

onde $P_{2}(t)$ é a probabilidade do sistema estar com o canal falho (ou seja, no estado 2) e a falha não ter sido detectada e $\tau_{p}$, o intervalo entre testes. A Eq.2 significa que os únicos eventos iniciadores de acidentes possíveis são os causados por demandas do sistema enquanto ele estiver falho e sua falha não tiver sido detectada, pois se assume que durante o reparo a planta está desligada (reparo offline).

\section{Método das Variáveis Suplementares}

Ainda que uma das taxas de transição seja função do tempo, o modelo será markoviano ao se inserir uma variável suplementar $(x)$, a idade do canal. O sistema de equações 
integro-diferenciais [3] será:

$$
\begin{gathered}
\frac{\partial p_{1}(x, t)}{\partial x}+\frac{\partial p_{1}(x, t)}{\partial t}=-\lambda(x) p_{1}(x, t) \\
\frac{d P_{2}(t)}{d t}=\int_{0}^{\infty} \lambda(x) p_{1}(x, t) d x-\nu P_{2}(t)+\gamma \mu P_{3}(t) \\
\frac{d P_{3}(t)}{d t}=\nu P_{2}(t)-\mu P_{3}(t)
\end{gathered}
$$

onde $p_{1}(x, t) \Delta x \Delta t$ é a probabilidade do sistema estar no estado 1 entre os instantes de tempo $t$ e $t+\Delta t$ e com idade entre $x$ e $x+\Delta x, \lambda(x)$ é a taxa de falha do sistema, dependente da sua idade e $t$ representa o tempo calendário. A probabilidade de erro humano é representada pela constante $\gamma$. A probabilidade do sistema se encontrar no estado 1 no instante $t$ será dada por:

$$
P_{1}(t)=\int_{0}^{\infty} p_{1}(x, t) d x
$$

Os estados apresentados na Fig.1 possuem os seguintes significados: o estado 1 (sistema em funcionamento) é modelado por meio da densidade de probabilidade $p_{1}(x, t)$, o estado 2 representa o sistema com o canal falho e sua falha não foi revelada, com probabilidade $P_{2}(t)$ e o 3 representa o sistema falho, com a falha revelada, com probabilidade $P_{3}(t)$.

A condição inicial para a Eq.3 é:

$$
p_{1}(x, 0)=f(x)
$$

onde $f(x)$ é a densidade de probabilidade dos tempos de falha do canal. Admitimos que os tempos de falha seguem uma distribuição Weibull de 3 parâmetros. Teremos para $\lambda(x)$ e $f(x)$, respectivamente:

$$
\lambda(x)=\left\{\begin{array}{cc}
\lambda_{0}, & x<x_{0} \\
\lambda_{0}+\left(\frac{m}{\theta}\right)\left(\frac{x-x_{0}}{\theta}\right)^{m-1}, & x \geq x_{0}
\end{array}\right.
$$

$\mathrm{e}$

$$
f(x)=\left\{\begin{array}{cl}
\lambda_{0} e^{-\lambda_{0} x}, & x<x_{o} \\
\left(\lambda_{0}+\left(\frac{m}{\theta}\right)\left(\frac{x-x_{0}}{\theta}\right)^{m-1}\right) e^{-\lambda_{0} x-\left(\frac{x-x_{0}}{\theta}\right)^{m}}, & x \geq x_{o}
\end{array}\right.
$$

onde $m$ é o parâmetro de forma e $m>1$, pois o sistema envelhece. $\theta$ é o parâmetro de escala, $\lambda_{0}$, a taxa de falha do sistema antes dele envelhecer e, portanto, constante e $x_{0}$, a idade do canal quando do início do seu envelhecimento. Além da Eq.7, necessitamos também das seguintes condições:

$$
\begin{gathered}
p_{1}\left(x_{0}, t\right)=(1-\gamma) \mu P_{3}(t) \\
P_{1}(0)=1 \\
P_{i}(0)=0, \quad \mathrm{i}=2,3
\end{gathered}
$$


A condição de contorno, Eq.10, mostra o estado do canal na idade $x_{0}$. As probabilidades $P_{1}\left(x_{0}\right), P_{2}\left(x_{0}\right)$ e $P_{3}\left(x_{0}\right)$ são conhecidas, pois antes do envelhecimento os tempos de falha são exponenciais. As Eq.11 e Eq.12 representam as probabilidades do sistema de proteção se encontrar em cada estado, 1, 2 ou 3, no início do funcionamento do mesmo, ou seja, em $t=0$. É admitido que neste instante o sistema está funcionando.

É claro que:

$$
\sum_{i=1}^{3} P_{i}(t)=1
$$

A partir da definição da Eq.2, nota-se que nos interessa apenas a probabilidade $P_{2}(t)$, pois como já mencionado, consideramos que se realizam somente reparos offline.

\section{Solução do Sistema de Equações}

O sistema de equações é acoplado. O método de solução escolhido foi o das diferenças finitas [4]. Para a solução numérica das integrais, foi usado o método de Simpson Composto [4]. O primeiro passo é definir a malha sobre a qual o método vai funcionar:

$$
\begin{gathered}
x_{1}=x_{0}, \quad x_{j+1}=x_{0}+j \Delta x, x_{j+1}-x_{j}=\Delta x ; \forall j=1, \cdots, J \\
t_{1}=0, t_{l+1}=l \Delta t, t_{l+1}-t_{l}=\Delta t ; \forall l=1, \cdots, L
\end{gathered}
$$

onde o índice $j$ se refere à variável suplementar e o $l$ representa o tempo calendário. Estendendo esta notação para as probabilidades, teremos:

$$
\begin{gathered}
p_{1}\left(x_{j}, t_{l}\right)=p_{1, j}^{l}, \forall j=1, \cdots, J+1 ; \quad l=1, \cdots, L+1 \\
P_{i}\left(t_{l}\right)=P_{i}^{l}, l=1, \cdots, L+1, \quad i=2,3
\end{gathered}
$$

Aplicando as condições de contorno, Eqs.7 e 10-12, teremos, respectivamente:

$$
\begin{gathered}
p_{1, j}^{1}=f_{j}, \forall j=1, \cdots, J+1 \\
p_{1,1}^{l}=(1-\gamma) \mu P_{3}^{l}, \quad \forall l=2, \cdots, L+1 \\
P_{1}^{1}=1 \\
P_{i}^{1}=0, \quad i=2,3
\end{gathered}
$$

Para a discretização da Eq.3, faremos a seguinte aproximação que pode ser justificada expandindo as duas probabilidade entre colchetes em séries de série de Taylor até os termos lineares e somando as expansões, membro a membro [4].

$$
p_{1, j}^{l} \simeq \frac{1}{2}\left[p_{1, j+1}^{l}+p_{1, j-1}^{l}\right]
$$

Para as derivadas parciais, temos:

$$
\frac{\partial p_{1}(x, t)}{\partial x} \simeq \frac{p_{1, j+1}^{l}-p_{1, j-1}^{l}}{2 \Delta x}
$$




$$
\frac{\partial p_{1}(x, t)}{\partial t} \simeq \frac{p_{1, j}^{l+1}-p_{1, j}^{l}}{\Delta t}
$$

Assim, substituindo a Eq.22 na Eq.24:

$$
\frac{\partial p_{1}(x, t)}{\partial t} \simeq \frac{p_{1, j}^{l+1}-\frac{1}{2}\left[p_{1, j+1}^{l}+p_{1, j-1}^{l}\right]}{\Delta t}
$$

Esta discretização é conhecida como método de Lax-Friedrichs e tem estabilidade condicionada [5]. Assim, a Eq.3 se tornará:

$$
p_{1, j}^{l+1}=-\lambda_{j} \Delta t p_{1, j}^{l}+\frac{1}{2}\left(1+\frac{\Delta t}{\Delta x}\right) p_{1, j-1}^{l}+\frac{1}{2}\left(1-\frac{\Delta t}{\Delta x}\right) p_{1, j+1}^{l}
$$

Esta equação é válida para $j=2, \cdots, J ; l=1, \cdots, L$. Já, para $j=J+1$, é utilizada a Eq.22 da seguinte forma:

$$
p_{1, J+1}^{l+1}=2 p_{1, J}^{l+1}-p_{1, J-1}^{l+1}
$$

Continuando, teremos então, para as derivadas:

$$
\frac{d P_{i}(t)}{d t} \simeq \frac{P_{i}^{l+1}-P_{i}^{l}}{\Delta t} ; \forall l=1, \cdots, L, i=2,3
$$

Na Eq. 4 temos de aproximar numericamente a integral. Como mencionado anteriormente, o método escolhido foi o de Simpson Composto [4], portanto teremos:

$$
\int_{0}^{\infty} \lambda(x) p_{1}(x, t) d x \simeq \frac{\Delta x}{3}\left[\lambda_{1} p_{1,1}^{l}+4 \sum_{j=1}^{\frac{J}{2}} \lambda_{2 j} p_{1,2 j}^{l}+2 \sum_{j=1}^{\frac{J}{2}-1} \lambda_{2 j+1} p_{1,2 j+1}^{l}+\lambda_{J+1} p_{1, J+1}^{l}\right]
$$

com J par. Substituindo a Eq.29 na Eq.4:

$$
P_{2}^{l+1}=P_{2}^{l}+\Delta t\left[-\nu P_{2}^{l}+\gamma \mu P_{3}^{l}+I\right]
$$

onde:

$$
I=\frac{\Delta x}{3}\left(\lambda_{1} p_{1,1}^{l}+4 \sum_{j=1}^{\frac{J}{2}} \lambda_{2 j} p_{1,2 j}^{l}+2 \sum_{j=1}^{\frac{J}{2}-1} \lambda_{2 j+1} p_{1,2 j+1}^{l}+\lambda_{J+1} p_{1, J+1}^{l}\right)
$$

Finalmente, para a probabilidade $P_{3}(t)$, temos, substituindo a Eq.28 na Eq.5:

$$
P_{3}^{l+1}=P_{3}^{l}+\Delta t\left[-\mu P_{3}^{l}+\nu P_{2}^{l}\right]
$$

Para encontrar $P_{1}(t)$ aplicamos o método de Simpson Composto à Eq.6:

$$
P_{1}^{l}=\frac{\Delta x}{3}\left(p_{1,1}^{l}+4 \sum_{j=1}^{\frac{J}{2}} p_{1,2 j}^{l}+2 \sum_{j=1}^{\frac{J}{2}-1} p_{1,2 j+1}^{l}+p_{1, J+1}^{l}\right)
$$


Para a Eq.3, fazendo $l=1$ teremos:

$$
\begin{gathered}
p_{1,1}^{1}=f_{1}, p_{1,1}^{2}=(1-\gamma) \mu P_{3}^{2} \\
p_{1, j}^{2}=-\lambda_{j} \Delta t p_{1, j}^{1}+\frac{1}{2}\left(1+\frac{\Delta t}{\Delta x}\right) p_{1, j-1}^{1}+\frac{1}{2}\left(1-\frac{\Delta t}{\Delta x}\right) p_{1, j+1}^{1}, \forall j=2, \cdots, J \\
p_{1, J+1}^{2}=2 p_{1, J}^{2}-p_{1, J}^{2}, j=J+1
\end{gathered}
$$

Dependendo dos valores de $\Delta t$ e $\Delta x$ adotados, a solução pode se tornar instável. Para este sistema de equações, o método é estável se $\frac{\Delta t}{\Delta x} \leq 1$ [5], obtida através da análise da Eq.26. Além desta condição, devemos ter também: $\Delta t \leq 1 / \nu$ e $\Delta t \leq 1 / \mu$, Eq.30 e Eq.32.

Podemos então calcular a frequência de ocorrência de acidentes, utilizando, novamente, o método de Simpson Composto:

$$
\eta=\frac{\nu}{\tau_{p}} \frac{\Delta t}{3}\left(P_{2}^{1}+4 \sum_{l=1}^{\frac{L}{2}} P_{2}^{2 l}+2 \sum_{l=1}^{\frac{L}{2}-1} P_{2}^{2 l+1}+P_{2}^{L+1}\right)
$$

\section{Resultados}

Os dados de entrada empregados são apresentados na Tabela 1. Os sem referência bibliográfica foram adotados baseados na nossa experiência.

O método foi testado para tempos exponenciais (comparação com os resultados de [1]) e, também, para o caso do comportamento assintótico em relação à idade $x$.

Tabela 1: -Valores utilizados para o cálculo dos atributos de interesse

\begin{tabular}{|c|c|c|c|}
\hline Parâmetro & Valor & Parâmetro & Valor \\
\hline$\nu$ & 10 ano $^{-1}[5]$ & $\theta$ & 1 ano \\
\hline$\mu$ & 52 ano $^{-1}[5]$ & $\Delta_{t}$ & $5 \times 10^{-5}$ \\
\hline$\gamma$ & 0,1 & $\Delta_{x}$ & $5 \times 10^{-5}$ \\
\hline$\tau_{p}$ & 1 ano $[5]$ & $x_{0}$ & 1 ano \\
\hline$\lambda_{0}$ & 1,0 ano $^{-1}[5]$ & $t_{L+1}$ & 2 anos \\
\hline$m$ & 2,5 & $x_{J+1}$ & 50 anos \\
\hline \multicolumn{4}{|r}{} \\
\hline
\end{tabular}

Tabela 2: -Valores utilizados para o cálculo dos atributos de interesse

\begin{tabular}{|c|c|c|}
\hline$\theta$ (anos) & $\nu$ (/ano $)$ & $\mu\left(\right.$ ano $\left.^{-1}\right)$ \\
\hline \multirow{3}{*}{10,0} & 0,5 & 0,07 \\
\cline { 2 - 3 } & 10,0 & 0,21 \\
\cline { 2 - 3 } & 100,0 & 0,22 \\
\hline \multirow{3}{*}{1,0} & 0,5 & 0,23 \\
\cline { 2 - 3 } & 10,0 & 0,93 \\
\cline { 2 - 3 } & 100,0 & 1,07 \\
\hline
\end{tabular}


Comparando as frequências de ocorrência de acidente obtidos (Tabela 2) com os de [1], os valores obtidos são razoáveis: para valores maiores de $\mathrm{m}$, os de $\eta$ também o são. $\mathrm{O}$ método retorna valores razoáveis para taxas de demanda de todas as faixas.

De acordo com o número de pontos com que se define a malha, $J$ e $L$, o tempo de cálculo cresce bastante, sendo $L$ o que mais o afeta. Para $L>10^{6}$, o tempo de cálculo é da ordem de $10 \mathrm{~h}$. Isto implica em se ponderar sobre o refinamento da malha, até se encontrar um ótimo, e a de se manter o intervalo do tempo calendário, $t$, curto. A otimização do tamanho da malha foi estabelecida para que o resultado da integral da densidade de probabilidade tivesse um erro menor que $10^{-10}$. Este valor foi escolhido por conta da precisão do método. Concluiu-se que para erros de ordem de grandeza maiores que $10^{-6}$ o método se tornava rapidamente instável. A escolha de erros de menor ordem de grandeza influencia o tempo de cálculo, uma vez que as integrais, que demandam maior esforço computacional, são todas em relação a $x$, ou seja, dependem de $J$. Os cálculos foram feitos em MATLAB v.2017.

\section{Conclusões}

O modelo desenvolvido pode servir como ferramenta para ajudar na tomada de decisões de políticas de reparo e extensão da vida útil de uma planta. A comparação do presente método com outros, por exemplo o dos estágios [3], pode também ser assunto de pesquisas seguintes. A aplicação de estudos de sensibilidade, por meio do Teoria Perturbação Generalizada [6], por exemplo, traria melhor entendimento do sistema e suas peculiaridades.

\section{Referências}

[1] L.F. Oliveira and J.D.A. Netto. Influence of the Demand Rate and Repair Rate on the Reliability of a Single-Channel Protective System. Rel. Eng., 17:267-276, 1987.

[2] L.F. Oliveira, R. Youngblood, P.F. Frutuoso e Melo. Hazard Rate of a Plant Equipped with a Two-Channel Protective System Subject to a High Demand Rate. Rel. Eng. Sys. Saf., 28:35-58, 1990.

[3] C.Singh, R. Billinton. System Reliability Modelling and Evaluation. Hutchinson, London, 1977.

[4] A.C. Alvim. Métodos Numéricos em Engenharia Nuclear, Ed. Certa, Curitiba, 2007.

[5] A.O Fortuna. Técnicas Computacionais para Dinâmica dos Fluidos: Conceitos Básicos e Aplicações. Edusp, São Paulo, 2000.

[6] E.F. Lima, D.G. Teixeira, P.F. Frutuoso e Melo, F.C. Silva, A.C.M. Alvim. Sensitivity Analysis of the Accident Rate of a Plant by the Generalized Perturbation Theory. Int. J. Math. Mod. and Met. in Applied Sciences, 10:309-316, 2016. 